\title{
BRIP1 loss-of-function mutations confer high risk for familial ovarian cancer, but not familial breast cancer
}

Nana Weber-Lassalle ${ }^{1 \dagger}$, Jan Hauke ${ }^{1 \dagger}$, Juliane Ramser ${ }^{2}$, Lisa Richters ${ }^{1}$, Eva Groß², Britta Blümcke ${ }^{1}$, Andrea Gehrig ${ }^{3}$, Anne-Karin Kahlert ${ }^{4,5}$, Clemens R. Müller ${ }^{3}$, Karl Hackmann ${ }^{4}$, Ellen Honisch ${ }^{6}$, Konstantin Weber-Lassalle', Dieter Niederacher ${ }^{6}$, Julika Borde ${ }^{1}$, Holger Thiele ${ }^{7}$, Corinna Ernst ${ }^{1}$, Janine Altmüller ${ }^{7,8}$, Guido Neidhardt ${ }^{1}$, Peter Nürnberg ${ }^{7,9}$, Kristina Klaschik', Christopher Schroeder ${ }^{10}$, Konrad Platzer ${ }^{11}$, Alexander E. Volk ${ }^{12}$, Shan Wang-Gohrke ${ }^{13}$, Walter Just ${ }^{14}$, Bernd Auber ${ }^{15}$, Christian Kubisch ${ }^{12}$, Gunnar Schmidt ${ }^{15}$, Judit Horvath ${ }^{16}$, Barbara Wappenschmidt ${ }^{1}$, Christoph Engel ${ }^{17,18}$, Norbert Arnold ${ }^{19}$, Bernd Dworniczak ${ }^{16}$, Kerstin Rhiem , Alfons Meindl ${ }^{2}$, Rita K. Schmutzler ${ }^{1}$ and Eric Hahnen ${ }^{1 *}$

\begin{abstract}
Background: Germline mutations in the BRIP1 gene have been described as conferring a moderate risk for ovarian cancer $(\mathrm{OC})$, while the role of BRIP1 in breast cancer $(\mathrm{BC})$ pathogenesis remains controversial.

Methods: To assess the role of deleterious BRIP1 germline mutations in BC/OC predisposition, 6341 wellcharacterized index patients with BC, 706 index patients with OC, and 2189 geographically matched female controls were screened for loss-of-function (LoF) mutations and potentially damaging missense variants. All index patients met the inclusion criteria of the German Consortium for Hereditary Breast and Ovarian Cancer for germline testing and tested negative for pathogenic BRCA1/2 variants.

Results: $B R I P 1$ LoF mutations confer a high OC risk in familial index patients (odds ratio $(O R)=20.97,95 \%$ confidence interval $(C l)=12.02-36.57, P<0.0001)$ and in the subgroup of index patients with late-onset $O C(O R=29.91,95 \%$ $C l=14.99-59.66, P<0.0001)$. No significant association of BRIP1 LoF mutations with familial BC was observed $(\mathrm{OR}=1.8195 \% \mathrm{Cl}=1.00-3.30, P=0.0623)$. In the subgroup of familial $\mathrm{BC}$ index patients without a family history of $\mathrm{OC}$ there was also no apparent association $(\mathrm{OR}=1.42,95 \% \mathrm{Cl}=0.70-2.90, P=0.3030)$. In 1027 familial $\mathrm{BC}$ index patients with a family history of $O C$, the BRIP1 mutation prevalence was significantly higher than that observed in controls $(\mathrm{OR}=3.59,95 \% \mathrm{Cl}=1.43-9.01 ; P=0.0168)$. Based on the negative association between BRIP1 LoF mutations and familial $\mathrm{BC}$ in the absence of an $\mathrm{OC}$ family history, we conclude that the elevated mutation prevalence in the latter cohort was driven by the occurrence of $\mathrm{OC}$ in these families. Compared with controls, predicted damaging rare missense variants were significantly more prevalent in $\mathrm{OC}(P=0.0014)$ but not in $\mathrm{BC}(P=0.0693)$ patients.
\end{abstract}

Conclusions: To avoid ambiguous results, studies aimed at assessing the impact of candidate predisposition gene mutations on BC risk might differentiate between BC index patients with an OC family history and those without. In familial cases, we suggest that BRIP1 is a high-risk gene for late-onset OC but not a BC predisposition gene, though minor effects cannot be excluded.

Keywords: Breast cancer, Ovarian cancer, BRIP1 gene, Germline mutations

\footnotetext{
* Correspondence: eric.hahnen@uk-koeln.de

${ }^{\dagger}$ Equal contributors

${ }^{1}$ Center for Familial Breast and Ovarian Cancer, Center for Integrated

Oncology (CIO), Medical Faculty, University Hospital Cologne, Kerpener

Straße 34, 50931 Cologne, Germany

Full list of author information is available at the end of the article
} 


\section{Background}

Monoallelic germline mutations in known predisposition genes, including BRCA1 (OMIM 113705) and BRCA2 (OMIM 600185), explain less than half of all cases of familial breast cancer $(\mathrm{BC})$ and/or ovarian cancer (OC), and confer moderate to high risk [1-6]. In contrast to mutations in BRCA1 and BRCA2, which are predisposing for both $\mathrm{BC}$ and $\mathrm{OC}$, several risk genes appear to be tumour-site specific. Inactivating CHEK2 (OMIM 604373) gene alterations predisposes to $\mathrm{BC}$ but not $\mathrm{OC}$ [6]. Conversely, women carrying deleterious RAD51C (OMIM 602774) germline mutations are at risk of developing $\mathrm{OC}$ [6] while an association with $\mathrm{BC}$ has not been established. Germline mutations in the BRIP1 (BRCA1interacting protein $\mathrm{C}$-terminal helicase 1) gene (OMIM 605882) have been reported to confer a moderate risk for $\mathrm{OC}$, especially the high-grade serous epithelial subtype [6-10], and prophylactic surgery is increasingly considered for BRIP1 mutation carriers [11]. The impact of germline BRIP1 mutations on $\mathrm{BC}$ risk, however, remains controversial.
BRIP1 was initially described as a $\mathrm{BC}$ predisposition gene in 2006 [12]. The analysis of 1212 women with familial BC and 2081 control individuals revealed heterozygous truncating mutations in nine index patients and two controls, resulting in a relative risk of 2.0 (95\% confidence interval $(\mathrm{CI})=1.2-3.2, P=0.012)$ for $\mathrm{BC}$. Buys et al. identified truncating BRIP1 mutations in 110 of 33,767 mainly familial $B R C A 1 / 2$-negative $\mathrm{BC}$ index patients, resulting in a cumulative carrier frequency of $0.33 \%$ [11], which is approximately two-fold higher than that described in the Exome Aggregation Consortium (ExAC) database (Table 1). Analysis of 1853 BRCA1/2negative index patients with familial $\mathrm{BC}$ by Easton et al. revealed similar results, not reaching levels of significance (odds ratio $(\mathrm{OR})=1.62,95 \% \mathrm{CI}=0.38-7.82, P=0.45$ ) $[10]$. Thompson et al. described comparable results in 2000 familial BRCA1/2-negative BC cases and 1997 controls $(\mathrm{OR}=1.75,95 \% \mathrm{CI}=0.51-5.99, P=0.55)$ [13]. Couch et al. reported that BRIP1 mutations confer a moderately increased risk of $\mathrm{BC}$ in 28,536 patients with familial and/or early-onset $\mathrm{BC}(\mathrm{OR}=1.63,95 \% \mathrm{CI}=1.11-2.41$,

Table 1 Prevalence of heterozygous loss-of-function germline mutations in the BRIP1 gene in control cohorts and BC/OC index patients according to tumour site, family history, and age at first diagnosis

\begin{tabular}{|c|c|c|c|c|c|c|c|}
\hline Study sample & $n$ & Negative & Positive (\%) & OR & $95 \% \mathrm{Cl}$ & $P$ value $^{a}$ & Mean AAD (range) \\
\hline ExAC control database & 27,173 & 27,135 & $38(0.14)$ & - & - & - & - \\
\hline FLOSSIES control database & 7325 & 7316 & $9(0.12)$ & - & - & - & - \\
\hline Geographically matched controls & 2189 & 2186 & $3(0.14)$ & - & - & - & - \\
\hline All controls & 36,687 & 36,637 & $50(0.14)$ & - & - & - & - \\
\hline OC index patients & 706 & 688 & $18(2.55)$ & 19.17 & $11.13-33.03$ & $<0.0001$ & $54(20-93)^{b}$ \\
\hline Affected by OC only & 523 & 507 & $16(3.06)$ & 23.12 & $13.08-40.88$ & $<0.0001$ & $53(20-93)^{c}$ \\
\hline Affected by OC and BC & 183 & 181 & $2(1.09)$ & 8.10 & $1.96-33.53$ & 0.0276 & $60(26-83)^{d}$ \\
\hline AAD OC $<51$ years & 246 & 244 & $2(0.81)$ & 6.01 & $1.45-24.82$ & 0.0471 & $39(20-50)$ \\
\hline AAD OC $<61$ years & 425 & 417 & $8(1.88)$ & 14.06 & $6.62-29.84$ & $<0.0001$ & $46(20-60)$ \\
\hline AAD OC $\geq 61$ years & 255 & 245 & $10(3.92)$ & 29.91 & $14.99-59.66$ & $<0.0001$ & $69(60-93)$ \\
\hline Familial OC index cases, overall & 611 & 594 & $17(2.78)$ & 20.97 & $12.02-36.57$ & $<0.0001$ & $54(20-93)^{\mathrm{e}}$ \\
\hline Familial OC index cases, relative(s) with BC only & 421 & 412 & $9(2.14)$ & 16.01 & $7.82-23.76$ & $<0.0001$ & $53(20-85)^{f}$ \\
\hline Familial OC index cases, relative(s) with OC & 190 & 182 & $8(4.21)$ & 32.21 & $15.06-68.90$ & $<0.0001$ & $54(21-93)^{9}$ \\
\hline$B C$ index patients & 6341 & 6325 & $16(0.25)$ & 1.85 & $1.06-3.26$ & 0.0363 & $47(17-92)$ \\
\hline$A A D B C<51$ years & 4417 & 4407 & $10(0.23)$ & 1.66 & $0.84-3.28$ & 0.1424 & $41(17-50)$ \\
\hline$A A D B C<61$ years & 5627 & 5612 & $15(0.27)$ & 1.96 & $1.10-3.49$ & 0.0272 & $44(17-60)$ \\
\hline$A A D B C \geq 61$ years & 714 & 713 & $1(0.14)$ & 1.03 & $0.14-7.45$ & 0.6260 & $68(61-92)$ \\
\hline Familial BC index cases, overall & 5668 & 5654 & $14(0.25)$ & 1.81 & $1.00-3.30$ & 0.0623 & $48(17-92)$ \\
\hline Familial BC index patients, relative(s) with BC only ${ }^{\text {h }}$ & 4641 & 4632 & $9(0.19)$ & 1.42 & $0.70-2.90$ & 0.3030 & $48(17-92)$ \\
\hline Familial BC index patients, relative(s) with $O C^{i}$ & 1027 & 1022 & $5(0.49)$ & 3.59 & $1.43-9.01$ & 0.0168 & $50(17-92)$ \\
\hline
\end{tabular}

Univariate logistic regression was performed to estimate odds ratios (OR) and $95 \%$ confidence interval (Cl)

$A A D$ age at first diagnosis, $B C$ breast cancer, ExAC Exome Aggregation Consortium, $O C$ ovarian cancer

${ }^{\mathrm{a}}$ Fisher's exact test

${ }^{b-g}$ Mean AAD and range refers to a subgroup of 680 patients $\left({ }^{b}\right), 511$ patients $\left({ }^{c}\right), 169$ patients $\left({ }^{d}\right), 593$ patients $\left({ }^{e}\right), 410$ patients $\left({ }^{f}\right)$, and 183 patients $\left({ }^{g}\right)$

${ }^{h} 2238 B C$ index patients had one relative with $B C$ and $2403 B C$ index patients had at least two relatives with $B C$

' All BC index patients reported at least one relative with OC. In addition, 382 BC index patients had no relatives with BC, $282 \mathrm{BC}$ index cases described one relative with $\mathrm{BC}$, and $363 \mathrm{BC}$ index patients two relatives with $\mathrm{BC}$ 
$P=0.01) \quad$ [14]. The elevated mutation prevalence points towards BRIP1 as a BC risk gene. This hypothesis is supported by the studies of Buys et al. and Couch et al., which showed that BRIP1 mutation prevalence was higher among women with triplenegative breast cancer (TNBC), a tumour phenotype associated with a hereditary disease cause $[11,15,16]$. In contrast to these findings, however, BRIP1 mutation analysis of 13,213 unselected patients with BC and 5242 control individuals by Easton et al. (SEARCH study) revealed no association with BC $(\mathrm{OR}=0.73,95 \% \mathrm{CI}=0.36-1.75, P=0.36) \quad$ [10]. Likewise, the recent analysis of Slavin et al. showed no association of truncating mutations in the BRIP1 gene with familial $\mathrm{BC}(\mathrm{OR}=0.60,95 \% \mathrm{CI}=0.10-2.33, P=0.77)$ [17]. In summary, the role of the BRIP1 gene in $\mathrm{BC}$ predisposition remains conflicting.

This case-control study aimed to further elucidate the role of BRIP1 in cancer predisposition by analysing its coding region (transcript NM_032043.2) in a wellcharacterized sample of $6341 \mathrm{BC}$ and 706 OC index patients of German descent, along with 2189 geographically matched female control individuals. We suggest that the elevated BRIP1 mutation prevalence described in some studies with the focus on familial $\mathrm{BC}$ might be due to the co-occurrence of $\mathrm{OC}$ in these families, one generally used criterion to define a positive cancer family history. To circumvent sample selection bias, we stratified our BC study sample by the familial occurrence of OC.

\section{Methods}

\section{Study cohorts}

All index patients were female and met the inclusion criteria of the German Consortium for Hereditary Breast and Ovarian Cancer (GC-HBOC) for germline testing (Additional file 1: Table S1). The GC-HBOC inclusion criteria are not restricted to familial cases and also consider patients with early-onset $\mathrm{BC}$ (age at first diagnosis (AAD) before 36 years), bilateral $\mathrm{BC}$ (AAD before 51 years), and patients affected by $\mathrm{BC}$ and $\mathrm{OC}$ even in the absence of a family history of $\mathrm{BC}$ and $\mathrm{OC}$ (Additional file 1: Table S1). Index patients with at least one relative affected by $\mathrm{BC}$ or $\mathrm{OC}$ were defined as familial index patients. Written informed consent was obtained from all patients, and ethical approval was granted by the Ethics Committee of the University of Cologne (07-048). All patients tested negative for pathogenic BRCA1/2 mutations. In addition, we analysed 2189 geographically matched female control individuals (GMCs). All GMCs were cancer-free and aged 40 years or above at the time of the blood draw (mean age $\pm S D, 63 \pm 10$ years). The cancer family history of the GMCs is undocumented. In this case-control investigation we additionally employed two publicly accessible control datasets (ExAC and
FLOSSIES). The FLOSSIES database includes gene panel sequence data of 7325 women of European American ancestry who are cancer-free until at least 70 years of age (cancer family history undocumented). The ExAC dataset comprises whole exome sequencing data of 27,173 individuals of non-Finnish European ancestry (excluding The Cancer Genome Atlas data). For ExAC, neither personal nor family cancer histories are publicly available.

\section{Next-generation sequencing}

All index patients and GMCs were screened for germline mutations in the BRIP1 gene (transcript NM_032043.2) by next-generation sequencing (NGS) using bloodderived DNA samples. NGS was performed at each participating centre using Illumina sequencing devices (MiSeq, NextSeq) employing either the customized TruRisk ${ }^{\bullet}$ (GC-HBOC designed; manufactured by Agilent or Illumina) or the TruSight ${ }^{\mathrm{Tm}}$ Cancer gene panel (Illumina) for hybrid capture target enrichment. Bioinformatic processing of the data was performed using JSI Medical Systems Sequence Pilot, Sophia Genetics DDM, or similar software packages certified for clinical diagnostics. The diagnostic pipelines of the laboratories involved have been successfully tested in European Molecular Genetics Quality Network (EMQN) schemes.

\section{Variant classification}

Variant classification was performed in accordance with the regulations of the international ENIGMA consortium (Evidence-based Network for the Interpretation of Germline Mutant Alleles; https://enigmaconsortium.org; version 1.1: 26 March 2015). All genetic variants were classified using a five-tier variant classification system as proposed by the International Agency for Research on Cancer (IARC) Unclassified Genetic Variants Working Group. All class 4/5 BRIP1 mutations identified by NGS were verified by standard Sanger sequencing. Truncating variants were defined as stop-gain, frameshift, or essential splice-site mutations affecting invariant splice sites or the last nucleotide of an exon. Loss-of-function (LoF) variants were defined as truncating variants not affecting the last exon of the BRIP1 gene (exon 20). For the identification of potentially damaging, rare missense variants we employed two in silico prediction tools (SIFT and MutationTaster). Missense variants were defined as potentially damaging when predicted deleterious by the in silico tools SIFT and MutationTaster (Alamut version 2.10 as 9 November 2017).

\section{Results}

All truncating mutations identified in the BRIP1 gene are listed in (Additional file 1: Table S2). The analysis of 706 OC index patients revealed 18 LoF mutation carriers, resulting in a cumulative carrier frequency of 
$2.55 \%$. Based on whole-exome sequencing data provided by the ExAC [18], 0.14\% of the individuals of non-Finnish European origin carried heterozygous LoF mutations within the BRIP1 gene (excluding The Cancer Genome Atlas data). This frequency is similar to that observed in the FLOSSIES database (https://whi.color.com). Of the 7325 women with American-European ancestry who remained cancer-free until at least 70 years of age, 9 (0.12\%) carried heterozygous BRIP1 LoF mutations. Among the 2189 GMCs screened in our study, 3 LoF mutation carriers were identified (cumulative carrier frequency, 0.14\%; Table 1). The comparison of the BRIP1 mutation prevalence in $\mathrm{OC}$ index patients and all controls revealed an OR of $19.17(95 \% \mathrm{CI}=11.13-$ 33.03, $P<0.0001$; Table 1).

In the overall cohort of $706 \mathrm{OC}$ index patients, 523 patients were affected by OC only and 183 by OC and BC. When stratified for personal cancer history, an OR of 23.12 (95\% CI $=13.08-40.88, P<0.0001$; Table 1 ) was observed in the subgroup of patients affected by OC only, which is considerably higher than the association observed in patients with $\mathrm{OC}$ and $\mathrm{BC}(\mathrm{OR}=8.10,95 \%$ $\mathrm{CI}=1.96 .08-33.53, P=0.0276$; Table 1$)$. The mean AAD of OC was reported for 680 out of 706 patients, with a mean of 54 years (range 20-93 years; Table 1). When stratified for AAD, the BRIP1 mutation prevalence rose with increasing AAD. While the association of BRIP1 LoF mutations with early-onset $\mathrm{OC}$ (AAD $<51$ years) was barely significant $(\mathrm{OR}=6.01,95 \% \mathrm{CI}=1.45-24.82$, $P=0.0471)$, a high OR of $29.91 \quad(95 \% \quad \mathrm{CI}=14.99$ 59.66, $P<0.0001)$ was observed in OC patients with an $\mathrm{AAD} \geq 61$ years. Of the $706 \mathrm{OC}$ index patients, 611 OC index patients show a $\mathrm{BC}$ and/or OC cancer family history. In familial OC index cases, an OR of 20.97 was observed (95\% CI $=12.02-36.57, P<0.0001$; Table 1). Of note, familial OC index patients with an additional OC family history show a considerably higher BRIP1 mutation prevalence $(\mathrm{OR}=32.21,95 \%$ $\mathrm{CI}=15.06-68.90, \quad P<0.0001$; Table 1$)$ than familial OC index patients with a BC-only family history (OR $=16.01,95 \% \mathrm{CI}=7.82-23.76, P<0.0001$; Table 1 ).

In summary, BRIP1 appears to be a high-risk gene for late-onset familial OC. All BRIP1 mutation carriers tested negative for further pathogenic mutations in $\mathrm{BC} / \mathrm{OC}$ predisposition genes (Additional file 1: Table S3). Most OC patients with BRIP1 mutations (mean AAD OC 61 years, range $26-76$ years) show a high-grade serous tumour phenotype (Additional file 1: Table S3), consistent with published results $[6,8]$. Importantly, the $\mathrm{OC}$ patient with an AAD of 26 years developed endometrioid OC, a comparatively rare OC subtype [19]. Following standard therapy, the patient remained cancer-free (last medical examination at 46 years). Large genomic rearrangements affecting the EPCAM gene were additionally excluded in this patient (data not shown).

In the overall sample of $6341 \mathrm{BC}$ index patients, 16 mutation carriers were observed, resulting in a cumulative carrier frequency of $0.25 \%(\mathrm{OR}=1.85,95 \% \mathrm{CI}=$ 1.06-3.26, $P=0.0363)$. Most mutation carriers with $\mathrm{BC}$ developed hormone receptor-positive $\mathrm{BC}$ (Additional file 1: Table S3). When stratified for AAD, a barely significant association of BRIP1 LoF mutations with BC was observed in the subgroup of patients with an AAD $<61$ years (Table 1). Of the $6341 \mathrm{BC}$ index patients, $5668 \mathrm{BC}$ index patients show a $\mathrm{BC}$ and/or $\mathrm{OC}$ cancer family history. No significant association between BRIP1 LoF mutations and familial $\mathrm{BC}$ was observed $(\mathrm{OR}=1.81,95 \%$ $\mathrm{CI}=1.00-3.30, P=0.0623$; Table 1$)$. In the $\mathrm{BC}$ sample comprising 4641 familial $\mathrm{BC}$ index patients without a personal or familial OC history, nine patients carried heterozygous BRIP1 LoF mutations, resulting in a cumulative carrier frequency of $0.19 \%(\mathrm{OR}=1.42,95 \% \mathrm{CI}=$ $0.70-2.90, P=0.3030$; Table 1). Analysis of 1027 familial $\mathrm{BC}$ index patients with a family history of $\mathrm{OC}$, however, revealed different results. In this cohort, we identified five patients carrying LoF mutations (cumulative carrier frequency, $0.49 \%$; Table 1 ). This frequency is significantly higher than that observed in controls $(\mathrm{OR}=3.59,95 \%$ $\mathrm{CI}=1.43-9.01, P=0.0168$; Table 1 ). Based on the overall negative association between BRIP1 LoF mutations and familial $\mathrm{BC}$, we conclude that this elevated mutation prevalence was mainly driven by the familial occurrence of OC, rather than by a predisposing role of BRIP1 mutations in $\mathrm{BC}$ pathogenesis.

Data on proven deleterious BRIP1 missense mutations are sparse. However, Ramus et al. demonstrated an association with OC for rare BRIP1 missense variants (minor allele frequency $(\mathrm{MAF})<1 \%$ ) that were predicted damaging by in silico tools such as SIFT and MutationTaster [8]. In controls (ExAC, FLOSSIES, GMCs), the cumulative carrier frequency for rare BRIP1 missense variants (MAF $<1 \%$ ) predicted damaging by both SIFT and MutationTaster was $1.32 \%$ (485 of 36,687; Additional file 1: Table S4). Compared with controls, rare BRIP1 missense variants predicted damaging by both tools were significantly more prevalent in OC patients $(2.83 \%, 20$ of $706 ; P=0.00139)$, but not in BC patients (Additional file 1: Table S4).

\section{Discussion}

To avoid ambiguous results, studies aimed at assessing the impact of candidate predisposing gene mutations on $\mathrm{BC}$ risk might differentiate between $\mathrm{BC}$ index patients with a family history of $\mathrm{OC}$ and those without. In this study, LoF mutations in the BRIP1 gene were not statistically associated with familial BC (Table 1). Although we analysed a large series of index patients with $\mathrm{BC}$, 
minor effects of BRIP1 mutations on $\mathrm{BC}$ risk cannot be fully excluded, and this requires further investigation.

In study samples selected for cancer family history, for example, the mutation prevalence of a risk gene is generally higher than in unselected cases. The AGO-TR-1 trial, for example, revealed pathogenic BRCA1/2 variants in 109 out of 523 unselected patients with OC (20.8\%), while 71 $B R C A 1 / 2$ mutation carriers were observed in the subgroup of 225 familial cases (31.6\%) [19]. In our study, mainly focussing on index patients with familial OC, the BRIP1 mutation prevalence (cumulative carrier frequency, $2.78 \%$; Table 1 ) is considerably higher than in studies focussing on unselected patients with OC. Norquist et al. [6] identified BRIP1 LoF mutations in 23 out of 1915 unselected patients with $\mathrm{OC}$ (cumulative carrier frequency, $1.20 \%)$. Kurian and colleagues [20] analysed 5020 patients with OC, most of whom were not showing a positive family history. In this unselected sample of OC patients, 36 BRIP1 mutation carriers were observed (cumulative carrier frequency, $0.72 \%)$. Ramus et al. [8] identified 30 truncating BRIP1 mutations in 3257 patients (cumulative carrier frequency, $0.92 \%$ ) and three truncating mutations in 3444 controls (cumulative carrier frequency, 0.09\%).

Age-dependent disease risks cannot be calculated solely based on case-control data generated in our study. For BRIP1 mutation carriers, Ramus et al. calculated a cumulative $\mathrm{OC}$ risk by age 80 of $5.8 \%$ ( $95 \% \mathrm{CI}=3.6-9.1 \%)$, possibly warranting risk-reducing salpingo-oophorectomy (RRSO) [8]. The highly significant associations shown in our study suggest that BRIP1 represents a high-risk gene for late-onset $\mathrm{OC}$, further supporting the notion that RRSO should be considered for BRIP1 mutation carriers. For BRIP1 mutation carriers, we observed a mean AAD of 61 years (range 26-76 years), which tended to be older than in the overall sample of familial OC index patients (mean AAD 54 years, range 20-93 years; Table 1). The mean AAD for BRIP1 mutation carriers described in our study was comparable with the data presented by Norquist et al. [6] (mean AAD 62 years, range 43-79 years) and Ramus et al. [8] (mean AAD 64, range 47-82 years).

This study has several limitations. The cohort of familial OC index patients is comparatively small, and results should be validated in larger studies focussing on familial OC patients. Moreover, we focussed on truncating variants not affecting the last exon of the BRIP1 gene. Truncating last exon variants in the BRIP1 gene, which may or may not impair protein function, were present in $0.06 \%$ of the ExAC controls (Additional file 1: Table S2), in one familial OC index patient $(0.16 \%)$, and in two familial $\mathrm{BC}$ index patients without OC family history $(0.04 \%)$. Thus, the inclusion of truncating last exon variants would marginally change the calculated ORs, but not our main conclusions.

\section{Conclusions}

BRIP1 LoF mutations confer a high OC risk in familial index patients $(\mathrm{OR}=20.97,95 \% \mathrm{CI}=12.02-36.57, P<$ $0.0001)$ and in the subgroup of index patients with lateonset $\mathrm{OC}(\mathrm{OR}=29.91,95 \% \mathrm{CI}=14.99-59.66, P<0.0001)$. No significant association between BRIP1 LoF mutations and familial $\mathrm{BC}$ was observed $(\mathrm{OR}=1.81,95 \% \mathrm{CI}=1.00$ $3.30, P=0.0623)$, although minor effects cannot be excluded. For OC, the highly significant associations shown in our study suggest that BRIP1 represents a high risk rather than a moderately penetrant predisposition gene, further supporting the notion that RRSO should be considered for BRIP1 mutation carriers.

\section{Additional file}

\begin{abstract}
Additional file 1: Table S1. Inclusion criteria of the German Consortium for Hereditary Breast and Ovarian Cancer (GC-HBOC) for $B R C A 1$ and BRCA2 germline testing. Table S2. Heterozygous protein-truncating mutations identified in the BRIP1 gene. Figure S1. Characterization of the C.507G > A variant within the BRIPI gene (rs876660937) on transcript level. Table S3. Genotypes and phenotypes of heterozygous BRIP1 mutation carriers identified within the BC/OC index patient cohorts. Table S4. Potentially damaging missense variants identified in the BRIP1 gene. (PDF $215 \mathrm{~kb}$ )
\end{abstract}

\begin{abstract}
Abbreviations
AAD: Age at first diagnosis; BC: Breast cancer; Cl: Confidence interval; ExAC: Exome Aggregation Consortium; GC-HBOC: German Consortium for Hereditary Breast and Ovarian Cancer; GMC: Geographically matched control individual; LoF: Loss-offunction; MAF: Minor allele frequency; NGS: Next-generation sequencing; OC: Ovarian cancer; OR: Odds ratio; RRSS: Risk-reducing salpingo-oophorectomy; SD: Standard deviation; TNBC: Triple-negative breast cancer
\end{abstract}

\section{Acknowledgements}

We are very thankful to all family members who participated in this study.

\section{Funding}

This study was supported by the German Cancer Aid (\#109076, \#70111850) and the Ministry for Innovation, Science and Research of the State of North Rhine-Westphalia (\#323-8.0302.16.02-132142). This publication was supported by LIFE — Leipzig Research Center for Civilization Diseases, Universität Leipzig. LIFE is funded by means of the European Union, by the European Regional Development Fund (ERDF), and by means of the Free State of Saxony within the framework of the excellence initiative. The funders had no role in study design, data collection and analysis, decision to publish, or preparation of the manuscript.

Availability of data and materials

The publicly available control datasets analysed during the current study are available in the FLOSSIES (https://whi.color.com) and ExAC

(http://exac.broadinstitute.org) repositories.

\section{Authors' contributions}

NWL, JH, JR, LR, EG, BB, AG, AKK, CRM, KH, EHo, KWL, DN, JB, HT, CEr, JA, GN, $P N, K K, C S, K P, S W G, W J, B A, G S, J H O, B W, N A, B D, K R$, and $A M$ performed the molecular genetic studies and/or analysed the data. AEV, CK, and CEn provided DNA samples and/or clinical/genetic data. NWL, JH, RKS, and EHa wrote the manuscript. EHa had full access to all the data in the study and takes responsibility for the integrity of the data and the accuracy of the data analysis. All authors read and approved the final manuscript. 


\section{Ethical approval and consent to participate}

Written informed consent was obtained from all patients and control individuals, and ethical approval was granted by the Ethics Committee of the University of Cologne (07-048)

\section{Consent for publication}

Not applicable.

\section{Competing interests}

The authors declare that they have no competing interests.

\section{Publisher's Note}

Springer Nature remains neutral with regard to jurisdictional claims in published maps and institutional affiliations.

\begin{abstract}
Author details
${ }^{1}$ Center for Familial Breast and Ovarian Cancer, Center for Integrated Oncology (ClO), Medical Faculty, University Hospital Cologne, Kerpener Straße 34, 50931 Cologne, Germany. ${ }^{2}$ Department of Gynaecology and Obstetrics, Klinikum rechts der Isar der Technischen Universität München, Munich, Germany. ${ }^{3}$ Department of Human Genetics, University Würzburg, Biozentrum, Würzburg, Germany. ${ }^{4}$ Institute for Clinical Genetics, Technische Universität Dresden, Dresden, Germany. ${ }^{5}$ Department of Congenital Heart Disease and Pediatric Cardiology, University Hospital Schleswig-Holstein, Campus Kiel, Kiel, Germany. ${ }^{6}$ Department of Obstetrics and Gynecology, Medical Faculty, Heinrich-Heine-University, Düsseldorf, Germany. ${ }^{7}$ Cologne Center for Genomics, University of Cologne, Cologne, Germany. ${ }^{8}$ Institute of Human Genetics, University of Cologne, Cologne, Germany. ${ }^{9}$ Cologne Excellence Cluster on Cellular Stress Responses in Aging-Associated Diseases, Cologne, Germany. ${ }^{10}$ Institute of Medical Genetics and Applied Genomics, University Hospital Tuebingen, Tuebingen, Germany. ${ }^{11}$ Institute of Human Genetics, University of Leipzig Hospitals and Clinics, Leipzig, Germany. ${ }^{12}$ Institute of Human Genetics, University Medical Center Hamburg-Eppendorf, Hamburg, Germany. ${ }^{13}$ Department of Obstetrics and Gynecology, Medical Faculty, University of UIm, UIm, Germany. ${ }^{14}$ Institute of Human Genetics, University of Ulm, Ulm, Germany. ${ }^{15}$ Institute of Human Genetics, Hannover Medical School, Hannover, Germany. ${ }^{16}$ Department of Gynecology and Obstetrics, University Clinics Muenster, Muenster, Germany. ${ }^{17}$ Institute for Medical Informatics, Statistics and Epidemiology (IMISE), University of Leipzig, Leipzig, Germany. ${ }^{18}$ LIFE — Leipzig Research Centre for Civilization Diseases, University of Leipzig, Leipzig, Germany. ${ }^{19}$ Institute of Clinical Molecular Biology, Department of Gynecology and Obstetrics, University Hospital Schleswig-Holstein, Christian-Albrechts-University of Kiel, Kiel, Germany.
\end{abstract}

Received: 21 September 2017 Accepted: 10 January 2018 Published online: 24 January 2018

\section{References}

1. Easton DF, Pharoah PD, Antoniou AC, Tischkowitz M, Tavtigian SV Nathanson KL, Devilee P, Meindl A, Couch FJ, Southey M, et al. Gene-panel sequencing and the prediction of breast-cancer risk. N Engl J Med. 2015; 372(23):2243-57.

2. Meindl A, Hellebrand H, Wiek C, Erven V, Wappenschmidt B, Niederacher D, Freund M, Lichtner P, Hartmann L, Schaal H, et al. Germline mutations in breast and ovarian cancer pedigrees establish RAD51C as a human cancer susceptibility gene. Nat Genet. 2010;42(5):410-4.

3. Antoniou AC, Casadei S, Heikkinen T, Barrowdale D, Pylkas K, Roberts J, Lee A, Subramanian D, De Leeneer K, Fostira F, et al. Breast-cancer risk in families with mutations in PALB2. N Engl J Med. 2014;371(6):497-506.

4. Neidhardt G, Hauke J, Ramser J, Gross E, Gehrig A, Muller CR, Kahlert AK, Hackmann K, Honisch E, Niederacher D, et al. Association between loss-offunction mutations within the FANCM gene and early-onset familial breast cancer. JAMA Oncol. 2016;3(9):1245-1248.

5. Kiiski II, Pelttari LM, Khan S, Freysteinsdottir ES, Reynisdottir I, Hart SN, Shimelis H, Vilske S, Kallioniemi A, Schleutker J, et al. Exome sequencing identifies FANCM as a susceptibility gene for triple-negative breast cancer. Proc Natl Acad Sci U S A. 2014;111(42):15172-7.

6. Norquist BM, Harrell MI, Brady MF, Walsh T, Lee MK, Gulsuner S, Bernards SS, Casadei S, Yi Q, Burger RA, et al. Inherited mutations in women with ovarian carcinoma. JAMA Oncol. 2016;2(4):482-90.
7. Rafnar T, Gudbjartsson DF, Sulem P, Jonasdottir A, Sigurdsson A, Jonasdottir A, Besenbacher S, Lundin P, Stacey SN, Gudmundsson J, et al. Mutations in BRIP1 confer high risk of ovarian cancer. Nat Genet. 2011;43(11):1104-7.

8. Ramus SJ, Song H, Dicks E, Tyrer JP, Rosenthal AN, Intermaggio MP, Fraser L, Gentry-Maharaj A, Hayward J, Philpott S, et al. Germline mutations in the BRIP1, BARD1, PALB2, and NBN genes in women with ovarian cancer. J Natl Cancer Inst. 2015;107(11). https://doi.org/10.1093/jnci/djv214. Print 2015 Nov.

9. Kanchi KL, Johnson K, Lu C, McLellan MD, Leiserson MDM, Wendl MC, Zhang Q, Koboldt DC, Xie M, Kandoth C, et al. Integrated analysis of germline and somatic variants in ovarian cancer. Nat Commun. 2014;5:3156.

10. Easton DF, Lesueur F, Decker B, Michailidou K, Li J, Allen J, Luccarini C, Pooley KA, Shah M, Bolla MK, et al. No evidence that protein truncating variants in BRIP1 are associated with breast cancer risk: implications for gene panel testing. J Med Genet. 2016;53(5):298-309.

11. Buys SS, Sandbach JF, Gammon A, Patel G, Kidd J, Brown KL, Sharma L, Saam J, Lancaster J, Daly MB. A study of over 35,000 women with breast cancer tested with a 25-gene panel of hereditary cancer genes. Cancer. 2017;123(10):1721-1730.

12. Seal $S$, Thompson D, Renwick A, Elliott A, Kelly P, Barfoot R, Chagtai T, Jayatilake $\mathrm{H}$, Ahmed M, Spanova K, et al. Truncating mutations in the Fanconi anemia J gene BRIP1 are low-penetrance breast cancer susceptibility alleles. Nat Genet. 2006;38(11):1239-41.

13. Thompson ER, Rowley SM, Li N, Mclnerny S, Devereux L, Wong-Brown MW, Trainer AH, Mitchell G, Scott RJ, James PA, et al. Panel testing for familial breast cancer: calibrating the tension between research and clinical care. J Clin Oncol. 2016;34(13):1455-9.

14. Couch FJ, Shimelis H, Hu C, Hart SN, Polley EC, Na J, Hallberg E, Moore R, Thomas A, Lilyquist J, et al. Associations between cancer predisposition testing panel genes and breast cancer. JAMA Oncol. 2017;3(9):1190-1196.

15. Stevens KN, Vachon CM, Couch FJ. Genetic susceptibility to triple-negative breast cancer. Cancer Res. 2013;73(7):2025-30.

16. Couch FJ, Hart SN, Sharma P, Toland AE, Wang X, Miron P, Olson JE, Godwin AK, Pankratz VS, Olswold C, et al. Inherited mutations in 17 breast cancer susceptibility genes among a large triple-negative breast cancer cohort unselected for family history of breast cancer. J Clin Oncol. 2015;33(4):304-11.

17. Slavin TPMK, Lilyquist J, Vijai J, Neuhausen SL, Hart SN, Ravichandran V, Thomas T, Maria A, Villano D, Schrader KA, Moore R, Hu C, Wubbenhorst B, Wenz BM, D'Andrea K, Robson ME, Peterlongo P, Bonanni B, Ford JM, Garber JE, Domchek SM, Szabo C, Offit K, Nathanson KL, Weitzel JN, Couch FJ. The contribution of pathogenic variants in breast cancer susceptibility genes to familial breast cancer risk. NPJ Breast Cancer. 2017;3:22.

18. Lek M, Karczewski KJ, Minikel EV, Samocha KE, Banks E, Fennell T, O'DonnellLuria AH, Ware JS, Hill AJ, Cummings BB, et al. Analysis of protein-coding genetic variation in 60,706 humans. Nature. 2016;536(7616):285-91.

19. Harter PHJ, Heitz F, Reuss A, Kommoss S, Marmé F, Heimbach A, Prieske K, Richters L, Burges A, Neidhardt G, de Gregorio N, El-Balat A, Hilpert F, Meier W, Kimmig R, Kast K, Sehouli J, Baumann K, Jackisch C, Park-Simon TW, Hanker L, Kröber S, Pfisterer J, Gevensleben H, Schnelzer A, Dietrich D, Neunhöffer T, Krockenberger M, Brucker S, Nürnberg P, Thiele H, Altmüller J, Lamla J, Elser G, du Bois A, Hahnen E, Schmutzler R. Prevalence of deleterious germline variants in risk genes including BRCA1/2 in consecutive ovarian cancer patients (AGO-TR-1). PLoS One. 2017;12(10):e0186043.

20. Lincoln SE, Kobayashi Y, Anderson MJ, Yang S, Desmond AJ, Mills MA, Nilsen GB, Jacobs KB, Monzon FA, Kurian AW, et al. A systematic comparison of traditional and multigene panel testing for hereditary breast and ovarian cancer genes in more than 1000 patients. J Mol Diagn. 2015;17(5):533-44. 